\title{
Development and Implementation of a Concept for the Meta Description of Highway Driving Scenarios with Focus on Interactions of Road Users
}

\author{
Raphael Pfeffer, Jingyu He and Sax Eric \\ Institute for Information Processing Technologies, Karlsruhe Institute of Technology, Engesserstr. 5, Karlsruhe, Germany
}

Keywords: $\quad$ Automated Driving, Scenario based Testing, Meta Model, Simulation Methods, Scenario Classification.

Abstract: $\quad$ Nowadays reducing the individual risk for advanced driver assistant systems (ADAS) and automated driving while guaranteeing the overall safety on the highway remains a big challenge. The identification of corner test cases and driving scenarios is key in the development process but is still not entirely solved. In the past, many contributed to a unified scenario definition but often with different application focus. In this paper, we develop a new scenario meta model based on existing definitions serving a development and test process where the test data is captured in real (test) drives and its contained scenarios are derived. We present the novelty of our scenario model describing the behaviour of dynamic objects in highway situations and show a first application of our model and results calculating the uniqueness of scenarios using auto-encoders.

\section{INTRODUCTION}

With the rising public demand on the intelligent and safe driving functionality, more and more key innovations in the automotive domain include increasingly autonomous features appeared in the recent decades, especially under the conditions of driving on the highway, like Adaptive Cruise Control (ACC) in 1997 (Winner et al., 2012) and the first Lane Keeping Support (LKS) system in a Nissan production car in 2001 (Kawazoe et al., 2001). Bearing this in mind, many questions arise, such as how to verify upcoming automated driving functions and how to guarantee sufficiency of the verification and validation process, if a significant test coverage must be achieved (Langner et al., 2018). To test the automated vehicles, the widely used approach is driving millions of test kilometres in the real world. For example, Waymo announced in 2018 that its autonomous vehicles have driven more than 16 million kilometres on public roads in the United States (Alva, 2018). However, for highly automated driving functions, this approach is not a economically feasible method for a full validation as billions of kilometres are needed (Wachenfeld and Winner, 2016). Contrary to this statistical approach of physical test coverage, with purely simulation-based methods, a completeness of the required test coverage cannot be guaranteed.

Nevertheless, simulation methods offer advantages in terms of configurability of test cases and ef- ficiency in test execution. In this context, scenariobased testing has become a central concept for simulation-based XiL methods and beyond. Especially for the testing of driver assistance systems and highly automated driving functions, defined scenarios often serve as the basis for deriving relevant test cases for the system-under-test (Otten et al., 2018).

However, even scenario-based testing does not answer the question of the completeness of the test coverage, since the prior requirements cannot be derived in the laboratory for highly automated driving functions. Therefore, in our research work a concept for a new scenario meta-description is developed. It is applicable to use recorded data from series or fleet vehicles or databases from previous recordings (Figure $1)$.

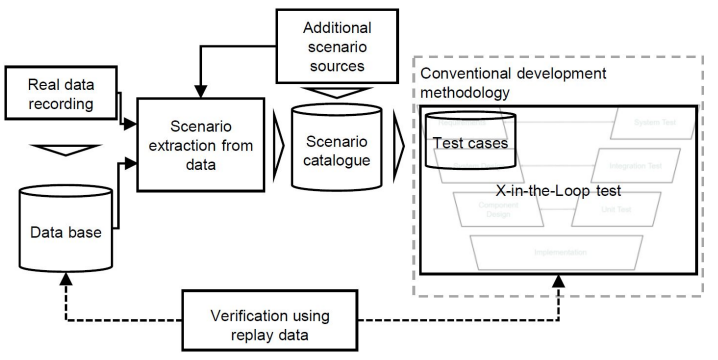

Figure 1: Process for real data based validation using simulation methods according to (Pfeffer et al., 2019).

This contribution focuses on a description model for the interaction with other road users and shows the 
application of machine learning techniques using this model data with a first target to discover the similarity between random scenarios.

The paper is organized as follows. Section II presents the previous works on defining the testing scenarios based on different application cases. Section III illustrates the architecture of the matrices, which can describe the scenario with the focus on the interaction with other road users on a highway. Sections IV presents the implementation of the scenario model in a simulation environment and the statistical results of the generated scenario matrices. Section V shows the first approach on how the matrices can be used to classify highway scenarios.

\section{RELATED WORK}

To test functionality, reliability and maturity of advanced driver assistance system and automated driving functions many achievements have been made in establishing scenario-based testing throughout the development process in the recent years.

\subsection{Scenario based Testing}

In 2014 Ulbrich et al. discuss different approaches of context modelling for automated driving scenarios in urban environments and analyze various context entities and layers in this approach. In this work scene is defined as a relationship between the context entities in the system architecture of automated vehicles. A scene can be taken as a link between vehicle guidance module and perception module whereas a situation refers to a central interface in vehicle guidance (Ulbrich et al., 2014).

Geyer et al. propose a metaphor-based terminology that consists of scenario, situation, scenery and scene of vehicles (Geyer et al., 2014). A driving scenario of a vehicle is metaphorized as a theatre. Scenery, as a structured collection of static elements, allows the director to create an environment suitable for the scene through different combinations, it consists of the basic geometry with predefined road types (intersection, country road, a motorway with exit and access, etc). Adding the dynamic elements, ego vehicle (considered system) and instructions they become a situation. A scenario consists of a series of situations (Figure 2).

Based on these works Ulbrich summarizes the definitions and substantiations of the terms scenario, situation and scene in the product life-cycle of automated vehicles and further illustrates the term scenario in the context of the V-model and the ISO26262

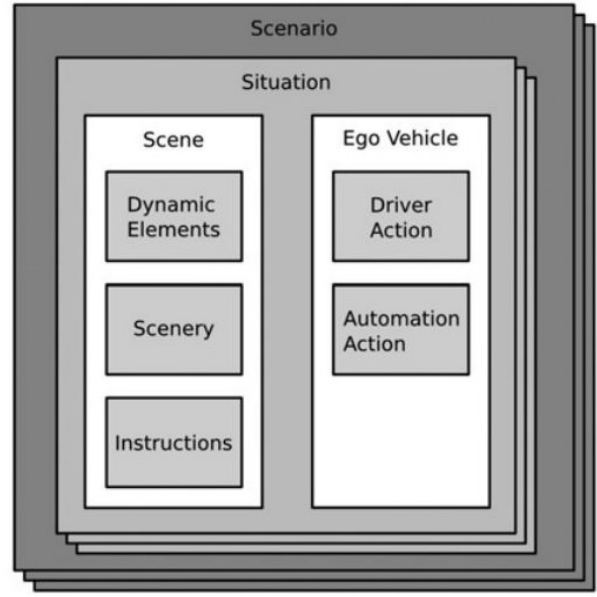

Figure 2: Metaphorical scenario concept according to (Geyer et al., 2014).

standard development process (Ulbrich et al., 2015). To be specific, this is intended by use-case and development-phase dependent levels of abstractions.

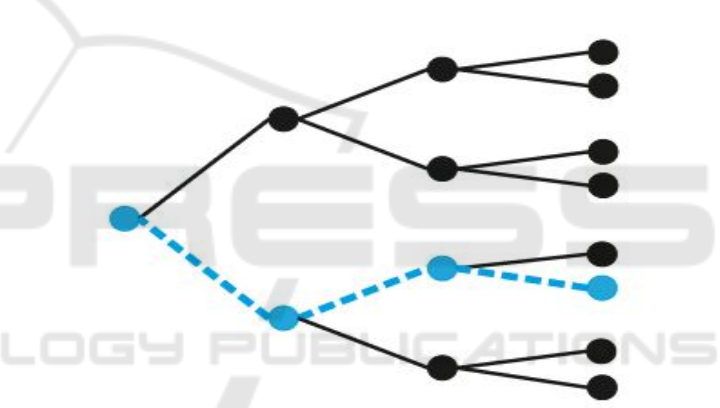

Figure 3: A scenario (dashed blue) as a temporal sequence of actions/events (edges) and scenes (nodes) (Ulbrich et al., 2015).

In this consideration, the term scenario can be understood as some kind of storyline, which can consist of at least one scene, action (or events) and goals (or values). The authors suggest the following definition for a scenario:

"A scenario describes the temporal development between several scenes in a sequence of scenes. Every scenario starts with an initial scene. Actions/events as well as goals/values may be specified to characterize this temporal development in a scenario. Other than a scene, a scenario spans a certain amount of time."(Ulbrich et al., 2015)

The principle of a scenario representation according to this definition is illustrated in Figure 3.

Another description of the scenario concept is proposed by Schuldt (Schuldt, 2017) in 2017 based on (Schuldt et al., 2015) and (Schuldt et al., 2018). Based 
on the previous developments to the scenario term, Schuldt introduces a test method, which is called a scenario-based testing approach. In this work a 4 layer model for a testing scenario is developed which contains different description layers for road geometry and topology, situation-specific adaptation of the road, environment and actors in which the previous scenario definitions are integrated. Compared with (Ulbrich et al., 2015), actions and goals are specified in detail and summarized together with the dynamic elements as the maneuvers.

Taking into account different requirements for the representation of scenarios in various process steps and aiming for a human-understandable scenario notation, in the context of the PEGASUS-project Menzel et al. discuss a detailed scenario description to design, development and testing automated vehicles in 2018 (Menzel et al., 2018). As evidenced by their self-reported survey results, the term "scenario" has not been defined uniformly, which makes it difficult to achieve a consistent understanding regarding the role of scenarios in the development process. Therefore, three abstract levels of the scenario are proposed, namely, functional level, logical level and concrete level (Menzel et al., 2019). While functional scenarios represent a level at which relevant properties are documented and described in language, logical scenarios define parameter areas in the state space for these properties in the form of entities and scenario relationships. Concrete scenarios are actual characteristics of a logical scenario, meaning that they represent a fixed parameter set. Logical scenarios can therefore be converted into at least one concrete scenario.

In the same year Steimle et al. propose a structure for a scenario terminology in the context of scenariobased testing using UML diagrams (Steimle et al., 2018b). In (Steimle et al., 2018a), their theory is further extended with three abstract layers combined with the scenario described in (Menzel et al., 2018). At this point the common general definition of scenario and its elements have stabilized so far although there is still no unifying standard.

\subsection{Scenario Meta Formats}

Scenarios and generated test cases must be structured in a generic way to be able to describe scenario content in different environments, such as highways, country roads or city streets, and to perform them with different test tools. Ideally, the structure can support the standardized usage of scenarios to seamlessly perform the scenarios throughout a variety of test tools, such as simulation. Therefore, it must allow existing test cases to be adapted to new or modified test objects. How can scenarios be represented technically to fulfil the requirements on various layers of abstraction with a standard format?

The OpenScenario project tries to answer this question. OpenScenario is an open file format for the description of dynamic contents in driving simulation applications developed by ASAM (Association for Standardization of Automation and Measuring Systems $)^{1}$. As a tool independent open file format, OpenScenario specifies the time-variant behaviour of entities during one simulation. State changes of traffic participants, infrastructure and other dynamic objects are described as actions triggered by conditions (PEGASUS-project, 2018). Due to the powerful language range connected with the OpenDrive (VIRES Simulationstechnologie $\mathrm{GmbH}, 2015$ ), complex traffic situations from the highway to urban areas can be described. OpenScenario project hat so far not been completed, but as an open description format it is currently widely used in research and industry.

For the description of dynamic components in a scenario different further approaches of semantic models exist. Exemplary, Wang et al. propose a new description for traffic element semantic features (Wang et al., 2018). In this work the global position of ego vehicle and traffic elements are combined and transformed to the relative distances and angles and are described as a matrix of traffic elements in each scene. Kohlhaas et al. present a further concept for the structured mapping of relations between the ego vehicle and other road users as well as the environment (Kohlhaas et al., 2014). The concept also tries to take into account the dynamics of the connections and formalizes rules for the transitions of the dynamic objects between the different states, which lead to new possible configurations or exclude invalid configurations. Petrich et al. extend this concept and design a compact semantic tensor representation to describe the relationships of all road users of a scenario in pairs (Petrich et al., 2018).

\section{NEW META MODEL FOR HIGHWAY APPLICATIONS}

Inspired by these approaches and following the requirement that the data must be derivable by a (sensor) record (see Figure 1), a model for the state and scene representation of the ego vehicle in relation to the surrounding traffic objects is suggested in this work. The spatial relations result in a possible dis-

\footnotetext{
${ }^{1}$ https://www.asam.net/standards/detail/openscenario/
} 
cretization for scenes on a highway to three-state possibilities each in longitudinal and lateral direction.

Besides, we propose an action model based on the driving maneuvers for the transitions between the scenes for all dynamic objects. This allows a scenario in the sense of the previously presented definitions to be modeled. The main elements will be presented in the following subsections.

\subsection{Matrix Representation for the Scene Description}

At first, the relevant highway section depending on the region of interest is divided into blocks using a simple grid model.

The positions of all dynamic objects are captured and longitudinally and laterally encoded relative to the ego vehicle along the road surface. The scene is divided laterally according to the lanes. Longitudinally we assume a maximum observation radius of $200 \mathrm{~m}$ and define the state "same level" at a maximum relative distance of $10 \mathrm{~m}$ to the ego vehicle (see Figure 4).

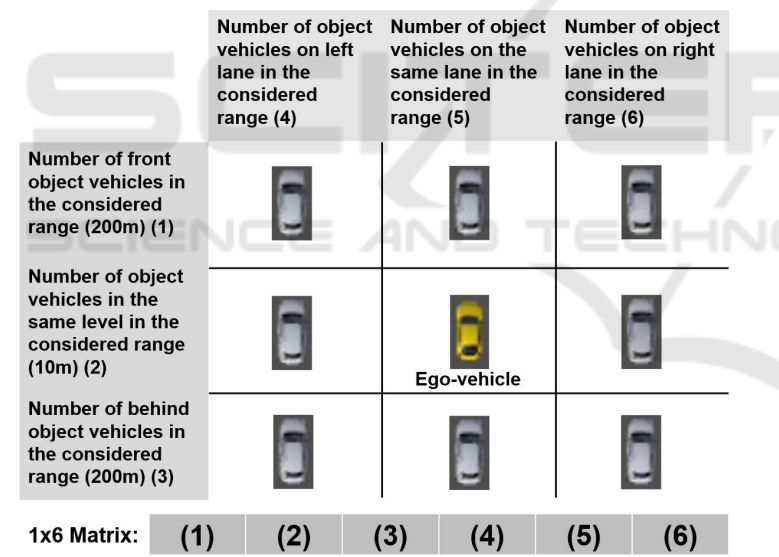

Figure 4: Matrix for the scene description.

In doing so, each of the dynamic objects, that appears surrounding the ego vehicle are taken into account of the scene description. We use an $1 * 6$ matrix where the first three digits represent the count of object vehicles that longitudinally appear in the front, same level and behind the ego vehicle. The last three digits indicate the number of object vehicles that laterally are located in the left lane, the same lane and the right lane compared to the ego vehicle.

For example, if two object vehicles are longitudinally in the behind position to the ego vehicle, then the first three digits of the $1 * 6$ matrix should be 0,0 and 2. At the same time, one of the two object vehicles is on the left lane and the other is on the right lane, as a result, the last three digits of the $1 * 6 \mathrm{ma}-$ trix should be 1,0 and 1 . So the whole $1 * 6$ matrix description should be $[0 ; 0 ; 2 ; 1 ; 0 ; 1]$.

\subsection{Scenario Meta-description Model}

Sequences between two scenes (as quasi static snapshots) are determined by the included actions and maneuvers (see Figure 3). In our work we determined so-called basic maneuvers (e.g. lane change left for all dynamic objects which allow clear transitions between the scenes respectively the depicted scenes abstraction. To describe the characteristics precisely, additional parameters are recorded. For longitudinal maneuvers, these are, for example, the initial and final velocity, the duration and the distance. Lateral maneuvers are described by the initial position and final lateral position as well as the duration of the maneuver. The maneuvers and their parameters are captured in a matrix representation analogous to the presented scene representation.

To merge the information for the dynamic progression of a scenario we use a tensor with three dimensions: time, objects, and meta information. Meta information contains the abstractions for the scenes and actions previously presented. These are recorded for all objects along a (recorded) scenario sequence. Along the time vector, time stamps of scene changes and action/maneuver changes are stored (Figure5).

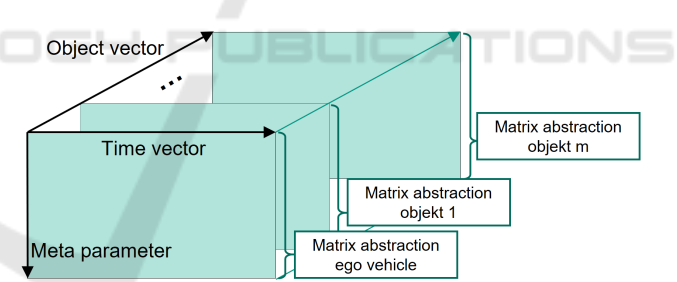

Figure 5: Principle of scenario meta information tensor for dynamic objects.

\subsection{Scenario Selection}

The scenario description model presented allows the storage of records of any length according to the process described in Figure 1. The question arises, how long should the scenarios actually be? As the current state of the art cannot provide a clear answer to this question, the application possibilities in our meta model are also kept flexible by its structure. Thus, scenarios of any length can be displayed with the tensor model (or be extracted from it). For example, a "cut-in vehicle" scenario could be represented by two scenes, while a scenario "overtaking" is possibly described by 3-4 consecutive scenes (see also Figure 8 . Another advantage is that even scenarios of different 
lengths of time can be compared if they belong to the same scenario class (e.g. "overtaking") since they still have the same tensor representation length. Figure 6 shows an example for two consecutive scenes (as part of an e.g. "overtaking" scenario) and its scene matrix representation. The $1 * 12$ scenario matrix means the chronological connection of two $1 * 6$ scene matrices and represents the transformation of different object vehicle distributions in a scenario with two scenes.

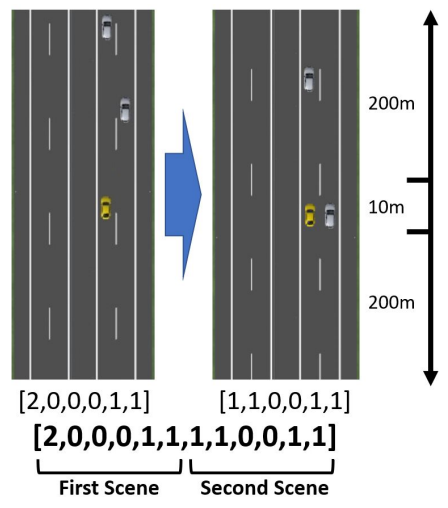

Figure 6: Matrix description for a scenario with two scenes.

\section{IMPLEMENTATION IN SIMULATION}

To identify the technical feasibility and adopt an engineering assessment, the scenario tensors in the development process can be extracted from real highway driving. In this stage of our work the data is first generated in a virtual driving simulation. As a replacement for a real test drive, the simulation software CarMaker is used. IPG CarMaker is a test platform which allows to create real-world test scenarios in a virtual environment, simulating every type of road and traffic, and performing realistic execution through an event and maneuver-based testing method (IPG Automotive $\mathrm{GmbH}, 2019)$. CarMaker provides models for vehicle with its sub-components, sensors, driver, road and many surrounding elements. All models and elements can be changed to generate a different behavior. Various of virtual sensors can be mounted on the ego vehicle model representing a vehicle with ADAS or automated driving functions (Figure 7).

The relevant information can be extracted using those sensors. For example, distance, relative speed and relative accelerations to all surrounding objects within the sensor range can be calculated. These data can also be used to calculate the grid positions according to our presented concept. Consequently, the overall scenario tensor is created as a result of each simu-

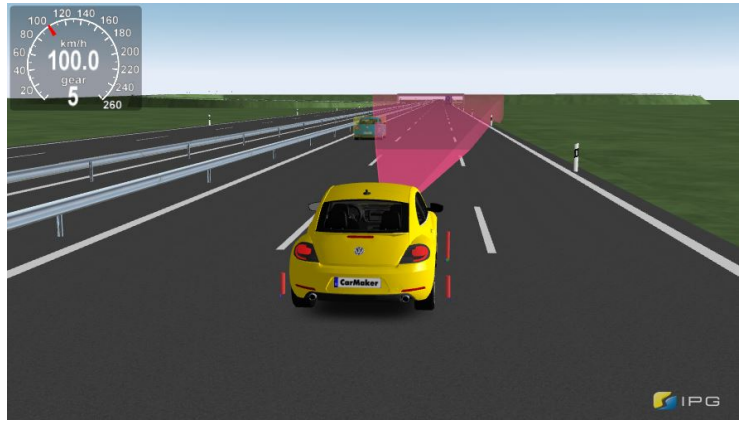

Figure 7: Vehicle model equipped with sensors in CarMaker and virtual highway environment.

Table 1: Test series in this work.

\begin{tabular}{|c|c|c|}
\hline Tests & Traffic density & Distance \\
\hline Test series 1 & 17.5 vehicles per $\mathrm{km}$ & $743.1 \mathrm{~km}$ \\
\hline Test series 2 & 7.5 vehicles per $\mathrm{km}$ & $181.9 \mathrm{~km}$ \\
\hline
\end{tabular}

lation run in a post-processing step. In this work, we created two highway test series with different highway cases (see Table 1). One simulates driving scenarios with crowded object vehicles, which represent slow moving traffic situations and jam. In this case we generated 128 autonomously driving objects on a 7.3 $\mathrm{km}$ two-lane highway, which means the traffic flow density is 17.5 vehicles per kilometer (test series 1). The other test series represents a lower traffic density situation of 7.5 vehicles per kilometer, which has 56 vehicles on the $7.3 \mathrm{~km}$ highway (test series 2). Based on these two basic tests we created random variations by manipulating parameters like cruising speed of ego vehicle, driver aggressiveness or the lane change behaviour of the dynamic objects. Finally, we run all variations and saved the ego vehicle's (sensor) data with a sample rate of 10 samples per second, from which the scenario meta information tensors were calculated. In total for the application presented here, we generated an overall length of 925 kilometers of highway driving data in the simulation.

\section{APPLICATION: SCENARIO SIMILARITY}

\subsection{Scenario Composition by Highway Maneuvers}

The scenario meta-description has a wide range of applications. The precise description of a feature from a whole scenario is among the first. Maneuvers are a certain pattern of the whole scenario (Schuldt, 2017). As maneuvers are defined as the transitions 
between two or more unique scenes we can use scene sequences to identify specific scenarios. Finally, our concept can be used to identify more complex maneuvers (than the basic maneuvers captured), maneuver sequences, or even specific scenarios in the metadescription. For this purpose we simply use the scene encodings in the following application. In this context, the concept can be used to classify and extract specific scenarios out of a time series which can be used for a particular unit-under-test. Figure 8 shows possible examples with its scenario meta-descriptions for four common scenarios on a highway.

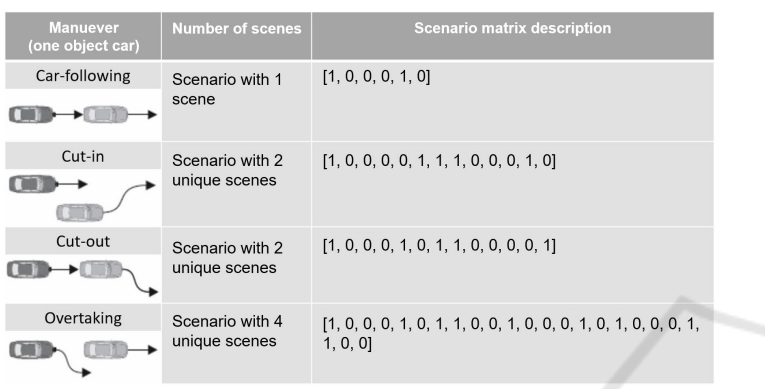

Figure 8: Scenario descriptions using scene matrix.

\subsection{Estimating the Uniqueness of Scenario Matrices}

In today's scenario-based testing for automated functions, a common approach is the manual scenario specification (Ulbrich et al., 2015) which can be extremely time-consuming if parameter boundaries are not considered properly. Some dedicate attention to critical situations and corner cases (Eckstein and Zlocki, ) but where a full test coverage cannot be achieved if the test cases generation happens without the consideration of real data.

To overcome these challenges we try to apply our scenario meta model to a test process where the data is derived from real test drives and the scenarios can be extracted automatically. Doing so, it is a necessity to be able to distinguish between new and so far unseen scenarios and known scenarios from e.g. former recordings. For an existing database of an arbitrary quantity of scenarios in terms of the interaction between vehicles, all new scenarios are supposed to be added to the database. For this reason the uniqueness of new scenarios has to be estimated. As a result, new (unique) scenario matrices provides no redundancy to the database. To achieve a higher test coverage for the function under test, test scenarios with new features and new content are added to the existing test scenario data pool. To solve this task we choose to apply the concept of auto-encoders as a machine learning technique for the detection of anomalies (here new sce- narios) in a first implementation which is presented as follows:

Auto-encoders are artificial neural networks with the ability to learn a compressed representation (encoding) for a set of data and thus also to extract essential features. Therefore, the similarity of external data to a training data set can be derived using the trained auto-encoder. While training the autoencoder, the trained model is validated with the validation data in each training epoch. To get the model with the best performance, the training hyperparameters, in this case batch size and learning rate, are adjusted and the average training error is compared after each training process. The Mean Square Error (MSE) is used as the reconstruction error.

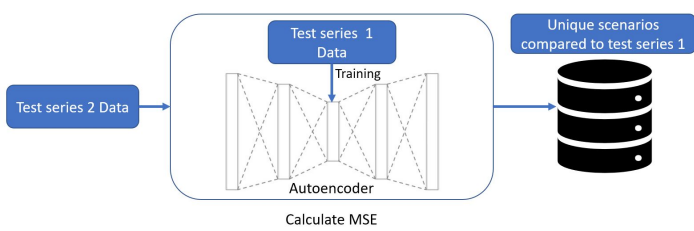

Figure 9: Calculation for uniqueness of scenario matrix using auto-encoders.

To ensure the uniqueness of the added data, scenario matrices that have a certain similarity degree to the existing scenarios are filtered and rejected. Figure 9 depicts the process to calculate the uniqueness of the test series 2 data and to filter the data that are similar to test series 1 . The scenario matrices from test series 1 in CarMaker are set as the training data and the data from the test series 2 are used to be detected and filtered. After the finished training, the models are applied on the scenario data of test series 2. The MSE as the reconstruction error is calculated for each sample, the results are then visualised to provide an intuitive impression.

A total of four auto-encoders is trained here to represent scenario descriptions consisting of one single scene up to four consecutive scenes. For the training data for each auto-encoder, the data set is split into training data and validation data with a ratio of $9: 1$. In the training data from test series 1 , scenario with one unique scene has 1439 samples, scenario with two unique scenes has 6312 samples, scenario with three unique scenes has 13302 samples and scenario with four unique scenes has 17666 samples.

Ultimately, a threshold for the reconstruction error for each type of scenario is subjectively determined and according to this threshold the scenarios in test series 2 are classified into two classes. Those with reconstruction errors below the threshold are regarded as known scenarios, while the others with reconstruction error above the threshold are regarded as unknown scenarios (anomaly scenarios). The classifi- 

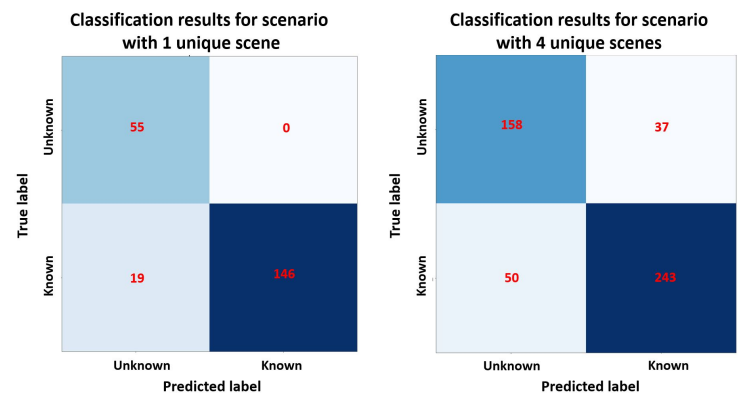

Figure 10: First results evaluation using confusion matrix.

cation model for unknown data detection is then evaluated by a confusion matrix.

Figure 10 describes the confusion matrix for scenarios with one unique scene in contrast to scenarios with four unique scenes. The results are evaluated in a confusion matrix in which each row of the matrix represents the scenarios in a predicted class while each column represents the scenarios in the ground truth. The visualization for the performance of the two classifications shows the marked difference in precision rate. With respect to the rate of positive predicted test series 2 data, the scenario matrices with one unique scene work better than scenario matrices with four unique scenes. The implementation achieves an accuracy at least for one unique scene scenarios where no unknown scenarios are falsely classified as known and thus rejected. This shows auto-encoders can be considered as an applicable method to classify unknown scenarios according to our meta model.

\section{FUTURE WORKS}

There are lots of additional challenges to be addressed with regards to the superior testing process we refer to in the introduction. Some ideas that would need further investigation are the following. A concept for the driving scenario meta-description is proposed in this contribution with its advantages as mentioned before. However, this concept in the current stage only considers the behaviour of the dynamic elements of a scenario. Many other features that can appear in a scenario e.g. traffic signs, further object properties, weather conditions and even the context of the total scenario are additional aspects which could be taken into account. In our future study, we will extend our concept and integrate more and more features into our meta model.

With regards to the auto-encoder, it has been shown that the auto-encoder is an efficient algorithm to reconstruct the features of a scenario. In this work auto-encoders with two hidden layers only have been used so far which do not seem deep enough to learn more features and fit larger data sets. In the future study we will extend the structure of auto-encoder so that it can reconstruct new scenarios more efficiently and achieve a better performance for classifying the known and unknown data from the existing scenario database.

The ultimate expectation for this research is to develop an integrated approach for efficient testing of automated vehicles. To validate the concept and its results so far it is needed to collect data from real tests instead of simulation and apply the presented methods in the future research.

\section{CONCLUSIONS}

In this paper, the state of the art for scenario definition is reviewed. We show a new approach for a scenario meta model with the focus on dynamic scenario elements which is suitable to be applied to a test process where the data is derived by physical test drives. An essential part of the scenario meta model is the representation of scenes which tries to solve the problem with time series data, where each sample has its duration by splitting the time series data into segments with the same state. Each scene segment is described in the form of a matrix of the same size independent of the actual time length so that the same type of scenario is encoded by the same size. By this approach scenarios of different recordings become comparable by the use of machine learning algorithms. We presented a first use case applied on our scenario meta model calculating the uniqueness of new scenarios compared with an existing data base with the help of auto-encoders.

Another advantage of this new scenario meta model is that the concept has a high versatility and can be extended easily. It is applicable to all features in a driving situation which can be recorded. For example, further properties like the weather conditions or traffic signs could also be integrated into the scene descriptions and therefore also be part of the scenario analysis. Last but not least, the concept has the potential to extend driving scenario descriptions to any period of time. At the end it should be possible to extract any sequences of scenes (scenarios) even out of recordings made over several hours.

\section{REFERENCES}

Alva, M. (2018). Waymo Self-Driving Cars Have Driven 16 Million Kilometres. [Online; accessed 01-July-2019]. 
Eckstein, L. and Zlocki, A. Safety potential of adas - combined methods for an effective evaluation. In The 23rd International Technical Conference on the Enhanced Safety of Vehicles (ESV) Seoul, Republic of Korea, May 27-30.

Geyer, S., Baltzer, M., Franz, B., Hakuli, S., Kauer, M., Kienle, M., Meier, S., Weißgerber, T., Bengler, K., Bruder, R., Flemisch, F., and Winner, H. (2014). Concept and development of a unified ontology for generating test and use-case catalogues for assisted and automated vehicle guidance. In IET Intelligent Transport Systems, volume 8, pages 183-189.

IPG Automotive GmbH (2019). CarMaker User's Guide (Version 8.0).

Kawazoe, H., Murakami, T., Sadano, O., Suda, K., and Ono, H. (2001). Development of a lane-keeping support system. In SAE Technical Paper. SAE International.

Kohlhaas, R., Bittner, T., Schamm, T., and Zöllner, J. M. (2014). Semantic state space for high-level maneuver planning in structured traffic scenes. In 17th International IEEE Conference on Intelligent Transportation Systems (ITSC), pages 1060-1065.

Langner, J., Bach, J., Ries, L., Otten, S., Holz, M., and Sax, E. (2018). Estimating the Uniqueness of Test Scenarios derived from Recorded Real-World-Driving-Data using Autoencoders. In 2018 IEEE Intelligent Vehicles Symposium (IV), number Iv, pages 1860-1866, Changshu, Suzhou, China.

Menzel, T., Bagschik, G., Isensee, L., Schomburg, A., and Maurer, M. (2019). From functional to logical scenarios: Detailing a keyword-based scenario description for execution in a simulation environment. In 2019 IEEE Intelligent Vehicles Symposium (IV), pages 2383-2390.

Menzel, T., Bagschik, G., Maurer, and Markus (2018). Scenarios for Development, Test and Validation of Automated Vehicles. In IEEE Intelligent Vehicles Symposium, Proceedings, volume 2018-June, pages 18211827.

Otten, S., Bach, J., Wohlfahrt, C., King, C., Lier, J., Schmid, H., Schmerler, S., and Sax, E. (2018). Automated assessment and evaluation of digital test drives. In Zachäus, C., Müller, B., and Meyer, G., editors, Advanced Microsystems for Automotive Applications 2017, pages 189-199, Cham. Springer International Publishing.

PEGASUS-project (2018). Basics for Testing - Stand 11: SCENARIO FORMATS. [Online; accessed 23-April2019].

Petrich, D., Azarfar, D., Kuhnt, F., and Zollner, J. M. (2018). The fingerprint of a traffic situation: A semantic relationship tensor for situation description and awareness. In 21 st IEEE International Conference on Intelligent Transportation Systems, ITSC 2018; Maui; United States; 4 November 2018 through 7 November 2018, pages 429-435. IEEE, Picataway (NJ).

Pfeffer, R., Schmidt, S., and Sax, E. (2019). Real data based validation of highly automated driving functions using simulation methods. ATZelectronics worldwide, 14(11):24-29.
Schuldt, F. (2017). Ein Beitrag für den methodischen Test von automatisierten Fahrfunktionen mit Hilfe von virtuellen Umgebungen. $\mathrm{PhD}$ thesis.

Schuldt, F., Maurer, M., and Menzel, T. (2015). Eine Methode fur die Zuordnung von Testfällen für automatisierte Fahrfunktionen auf X-in-the-Loop Verfahren im modularen virtuellen Testbaukasten. In 10. Workshop Fahrerassistenzsysteme., pages 171-182, Walting, Deutschland.

Schuldt, F., Reschka, A., and Maurer, M. (2018). A Method for an Efficient, Systematic Test Case Generation for Advanced Driver Assistance Systems in Virtual Environments, pages 147-175. Springer International Publishing, Cham.

Steimle, M., Bagschik, G., Menzel, T., Maurer, M., and Wendler, J. T. (2018a). Anwendung eines Grundvokabulars für den szenarienbasierten Testansatz automatisierter Fahrfunktionen anhand eines Beispiels. In AAET - Automatisiertes und vernetztes Fahren., Braunschweig, Deutschland.

Steimle, M., Bagschik, G., Menzel, T., Wendler, J. T., and Maurer, M. (2018b). Ein Beitrag zur Terminologie für den szenarienbasierten Testansatz automatisierter Fahrfunktionen. In AAET 2018 // AAET - Automatisiertes und Vernetztes Fahren, number September, pages 11-32.

Ulbrich, S., Menzel, T., Reschka, A., Schuldt, F., and Maurer, M. (2015). Defining and Substantiating the Terms Scene, Situation, and Scenario for Automated Driving. In 2015 IEEE 18th International Conference on Intelligent Transportation Systems, pages 982-988.

Ulbrich, S., Nothdurft, T., Maurer, M., and Hecker, P. (2014). Graph-based context representation, environment modeling and information aggregation for automated driving. In IEEE Intelligent Vehicles Symposium, Proceedings, pages 541-547.

VIRES Simulationstechnologie GmbH (2015). OpenDRIVE® Format Specification, Rev. 1.4. [Online; accessed 15-Feb-2020].

Wachenfeld, W. and Winner, H. (2016). The Release of Autonomous Vehicles, pages 425-449. Springer Berlin Heidelberg, Berlin, Heidelberg.

Wang, J., Zhang, C., Liu, Y., and Zhang, Q. (2018). Traffic Sensory Data Classification by Quantifying Scenario Complexity. In IEEE Intelligent Vehicles Symposium, Proceedings, volume 2018-June, pages 1543-1548.

Winner, H., Danner, B., and Steinle, J. (2012). Adaptive Cruise Control, pages 478-521. Vieweg+Teubner Verlag, Wiesbaden. 